Draft, November 23, 2017

Preprint typeset using $\mathrm{IATEX}_{\mathrm{E}}$ style emulateapj v. 12/16/11

\title{
SIMULATING THE GALAXY CLUSTER "EL GORDO”: GAS MOTION, KINETIC SUNYAEV-ZEL’DOVICH SIGNAL, AND X-RAY LINE FEATURES
}

\author{
Congyao Zhang ${ }^{1,2}$, QinguUan Yu ${ }^{2, \dagger}$, And Youjun Lu ${ }^{3,4}$ \\ ${ }_{1}$ Max Planck Institute for Astrophysics, Karl-Schwarzschild-Str. 1, D-85741 Garching, Germany \\ ${ }^{2}$ Kavli Institute for Astronomy and Astrophysics, Peking University, Beijing, 100871, China; ${ }^{\dagger}$ yuqj@pku.edu.cn \\ ${ }^{3}$ National Astronomical Observatories, Chinese Academy of Sciences, Beijing, 100012, China \\ ${ }^{4}$ School of Astronomy and Space Sciences, University of Chinese Academy of Sciences, No. 19A Yuquan Road, Beijing 100049, China \\ Draft, November 23, 2017
}

\section{ABSTRACT}

The massive galaxy cluster "El Gordo" (ACT-CL J0102-4915) is a rare merging system with a high collision speed suggested by multi-wavelength observations and the theoretical modeling. Zhang et al. (2015) propose two types of mergers, a nearly head-on merger and an off-axis merger with a large impact parameter, to reproduce most of the observational features of the cluster, by using numerical simulations. The different merger configurations of the two models result in different gas motion in the simulated clusters. In this paper, we predict the kinetic Sunyaev-Zel'dovich (kSZ) effect, the relativistic correction of the thermal Sunyaev-Zel'dovich (tSZ) effect, and the X-ray spectrum of this cluster, based on the two proposed models. We find that (1) the amplitudes of the kSZ effect resulting from the two models are both on the order of $\Delta T / T \sim 10^{-5}$; but their morphologies are different, which trace the different line-of-sight velocity distributions of the systems; $(2)$ the relativistic correction of the tSZ effect around $240 \mathrm{GHz}$ can be possibly used to constrain the temperature of the hot electrons heated by the shocks; and (3) the shift between the X-ray spectral lines emitted from different regions of the cluster can be significantly different in the two models. The shift and the line broadening can be up to $\sim 25 \mathrm{eV}$ and $50 \mathrm{eV}$, respectively. We expect that future observations of the $\mathrm{kSZ}$ effect and the X-ray spectral lines (e.g., by ALMA, XARM) will provide a strong constraint on the gas motion and the merger configuration of ACT-CL J0102-4915.

Keywords: galaxies: clusters: general - galaxies: clusters: individual (ACT-CL J0102-4915) - largescale structure of universe - methods: numerical - X-rays: galaxies: clusters

\section{INTRODUCTION}

Major mergers of galaxy clusters with relative velocity up to thousands of $\mathrm{kms}^{-1}$ can lead to violent disturbances in the density and the velocity fields of the intracluster medium (ICM). The line-of-sight motion of the gas in the ICM could be measured by the SunyaevZel'dovich (SZ) signal via the kinetic SZ (kSZ) effect (see a review in Birkinshaw 1999), and by the X-ray observations via the Doppler shitting and broadening of the spectral lines (Sunyaev et al. 2003; Biffi et al. 2013). These measurements are expected to provide insights into understanding the cluster merger configurations, the cosmic velocity field, and the gastrophysics of the ICM, though being a great challenge for observational techniques.

Over the past years, several efforts have been made to detect the $\mathrm{kSZ}$ signals via the statistical stacking method (Hand et al. 2012, Schaan et al. 2015, Planck Collaboration et al. 2016, Soergel et al. 2016) or observing the $\mathrm{kSZ}$ effect directly towards individual galaxy clusters (Mroczkowski et al. 2012; Sayers et al. 2013, 2016). One of the scientific goals for the present and the future cosmic microwave background (CMB) experiments is to map the kSZ effect resulting from large-scale structures (e.g., Benson et al. 2014). On the other hand, the new generation of X-ray instruments with unprecedented high spectral resolution is being developed to reveal the nature of gas motions in galaxy clusters, e.g., $X A R M$ (also known as ASTRO-H2), ATHENA.

In this work, we aim to predicting the observational features of the $\mathrm{kSZ}$ effect and the X-ray spectral lines of massive galaxy cluster ACT-CL J0102-4915 ("El Gordo") by using simulations. ACT-CL J0102-4915, first discovered by the Atacama Cosmology Telescope (ACT, see Marriage et al. 2011), is a rare merging system with a high collision speed (Menanteau et al. 2012 Jee et al. 2014). Its unique observational characteristics and inferred merging configurations make it an ideal target to investigate the motions of ICM during the cluster merging process. Zhang et al. (2015, hereafter ZYL15) have numerically explored the merger scenario of ACTCL J0102-4915, and proposed two models that can reproduce most of the features revealed by observations. In this paper, we present the simulated $\mathrm{kSZ}$ effect and the X-ray spectra for the two fiducial models in ZYL15, and show that future observations on these two aspects may help to constrain the merger configuration of ACT-CL J0102-4915.

This paper is organized as follows. In Section 2, we describe the method of simulating ACT-CL J0102-4915 and modeling its secondary $\mathrm{CMB}$ anisotropies and $\mathrm{X}$ ray spectra. Our main results, including the $\mathrm{kSZ}$ spatial distributions, the SZ spectra, relativistic correction of the tSZ effect, and the X-ray spectral lines for both of the fiducial models, are presented in Section 3. In Section 4 . we summarize our conclusions.

\section{METHOD}

2.1. The simulations 
ZYL15 have carried out a detailed study on identifying the merger configuration of ACT-CL J0102-4915 by using extensive numerical simulations, and found two fiducial models ( $\mathrm{A}$ and $\mathrm{B}$ ) that can match most of the observations of ACT-CL J0102-4915. Before describing the methodology used in this paper, we first summarize the simulations that have been performed in ZYL15 in this section.

ZYL15 perform simulations of mergers between two ideal galaxy clusters by adopting both the GADGET-2 code (Springel et al. 2001) and the FLASH code (Fryxell et al. 2000, Ricker 2008). Each cluster is simplified as a spherical halo consisting of collisionless dark matter and collisional gas, and the gas is assumed to be adiabatic. The merger parameters of the initial conditions include the mass of the primary (secondary) cluster $M_{1}\left(M_{2}\right)$, the mass ratio $\xi$ ( $\equiv M_{1} / M_{2}$ ), the gas fraction of the primary (secondary) cluster $f_{\mathrm{b} 1}\left(f_{\mathrm{b} 2}\right)$, the initial relative velocity $V$, and the impact parameter $P$. The parameter settings of fiducial models A and B are summarized in Table 1. These two models have different merger configurations: one is a nearly head-on merger with $P=300 h_{70}^{-1} \mathrm{kpc}$ (model A); the other is a highly off-axis merger with $P=800 h_{70}^{-1} \mathrm{kpc}$ (model B). These two models match the observations best (including the $\mathrm{X}$-ray luminosity and the temperature distributions, the SZ temperature decrement, the Mach numbers, etc.; see Table 2 in ZYL15) at $t=0.13$ and $0.14 \mathrm{Gyr}$ after the first pericentric passage, respectively. Model B can generate a remarkable wakelike substructure in the X-ray surface brightness map, like the one seen in the Chandra observation (Menanteau et al. 2012). Moreover, model B presents a larger radial relative velocity $\left(V_{\mathrm{r}}=1820 \mathrm{~km} \mathrm{~s}^{-1}\right)$ than model A $\left(V_{\mathrm{r}}=960 \mathrm{~km} \mathrm{~s}^{-1}\right)$, which, together with different impact parameters, is expected to be the main reason why these two models show different kSZ effects (see details in Section 3.1.

An extended model B, whose initial merger conditions are mostly the same as those in model B, is further proposed in ZYL15 to explore the effects of the gas density profile on the observed X-ray emission at the cluster outskirts. For simplicity, we focus on describing the effects of the above two fiducial models in this work, which is sufficient to illustrate the difference caused by their different impact parameters and radial velocities as to be seen below. Together with the results of the fiducial models, the results of extended model B will also be shown in the figures of this paper, which do not affect the main conclusion drawn from the comparison of the fiducial models.

In Section 2.2, we describe the method to model the secondary $\mathrm{CMB}$ anisotropies and the X-ray spectra for models A and B. The GADGET-2 and the FLASH simulations show little difference in the results for the purpose of this work. Unless specifically stated, all of the results below are based on the FLASH simulations presented in ZYL15.

\subsection{Mocking the secondary $C M B$ anisotropies and the $X$-ray spectra}

For any given merger snapshots, we can generate the maps of the secondary CMB anisotropies resulting from the cluster in the observer's sky plane, including the tSZ effect, the kSZ effect, and the Rees-Sciama (RS) effect by the following equations. In these equations, the subscripts "|l" and " $\perp$ " of a (position or velocity) vector are used to represent its components parallel and perpendicular to the line of sight, respectively.

- The changes in the CMB temperature at frequency $\nu$ by the tSZ effect can be obtained from the gas mass density $\rho_{\text {gas }}(\mathbf{r})$ and the temperature $T_{\text {gas }}(\mathbf{r})$ distributions by

$$
\left.\frac{\Delta T}{T_{\mathrm{CMB}}}\right|_{\mathrm{tSZ}} ^{\nu}=\frac{\sigma_{\mathrm{T}} k_{\mathrm{B}}}{m_{\mathrm{e}} c^{2}} \int n_{\mathrm{e}}(\mathbf{r}) T_{\text {gas }}(\mathbf{r}) \mathcal{F}\left(x_{\nu}, \Theta\right) \mathrm{d} \mathbf{r}_{\|},
$$

where $n_{\mathrm{e}}(\mathbf{r}) \equiv 0.9 \rho_{\text {gas }}(\mathbf{r}) / m_{\mathrm{H}}$ is the electron number density, and $T_{\mathrm{CMB}}, \sigma_{\mathrm{T}}, k_{\mathrm{B}}, m_{\mathrm{e}}, m_{\mathrm{H}}$, and $c$ represent the CMB temperature, the Thomson cross section, the Boltzmann constant, the electron mass, the hydrogen atom mass, and the speed of light, respectively. The tSZ distortion $\mathcal{F}\left(x_{\nu}, \Theta\right)$ is a function of $x_{\nu}\left(\equiv h \nu / k_{\mathrm{B}} T_{\mathrm{CMB}}\right)$ and $\Theta\left(\equiv k_{\mathrm{B}} T_{\text {gas }} / m_{\mathrm{e}} c^{2}\right)$. For the polynomial approximation, $\mathcal{F}\left(x_{\nu}, \Theta\right)=\sum_{i=0}^{i=4} Y_{i}\left(x_{\nu}\right) \Theta^{i}$ includes the relativistic correction (see eqs. 2.25-2.30 in Itoh et al. 1998), which, however, becomes less convergent when the gas temperature is higher $(\geq 30 \mathrm{keV}$, see more discussions in Itoh et al.1998 and Nozawa et al. 2000). The shock-heated gas in the simulations can reach a temperature higher than $50 \mathrm{keV}$ (see top panels in Fig. 6). To precisely determine the tSZ effect (particularly around the crossover frequency, $\nu_{0}$, defined by the frequency at which the tSZ effect vanishes), we adopt the numerical solution of the Boltzmann equation (see eqs. 2.12.5 in Itoh et al. 1998) as $\mathcal{F}\left(x_{\nu}, \Theta\right)$ in Equation (1). It is worth noting that our simulations model the ICM by using a single gas component, which implicitly assumes the electrons and ions reach the thermal equilibrium instantly. However, in reality this might not be the case. The electron-ion thermal equilibrium time-scale in the ICM is still poorly constrained (see Russell et al. 2012 and references therein). The tSZ effect at the shock region obtained from our simulations would be overestimated if the transport process between the electrons and ions is not sufficiently efficient. On the other hand, we find that the relativistic correction of the tSZ effect may be useful to give a constraint on the temperature of the shock-heated electrons, as to be demonstrated in Section 3.1.

- The changes in the CMB temperature at frequency $\nu$ by the $\mathrm{kSZ}$ effect is determined by

$$
\begin{aligned}
\left.\frac{\Delta T}{T_{\mathrm{CMB}}}\right|_{\mathrm{kSZ}} ^{\nu} & =\frac{\sigma_{\mathrm{T}} k_{\mathrm{B}}}{m_{\mathrm{e}} c^{2}} \int n_{\mathrm{e}}(\mathbf{r}) \times \\
& \left\{-\boldsymbol{\beta}_{\|} \cdot\left(\mathbf{r}_{\|} /\left|\mathbf{r}_{\|}\right|\right)\left[1+C_{1}\left(x_{\nu}\right) \Theta+C_{2}\left(x_{\nu}\right) \Theta^{2}\right]+\right. \\
& |\boldsymbol{\beta}|^{2}\left[\frac{1}{3} Y_{0}\left(x_{\nu}\right)+\left(\frac{5}{6} Y_{0}\left(x_{\nu}\right)+\frac{2}{3} Y_{1}\left(x_{\nu}\right)\right) \Theta\right]+ \\
& \left.\frac{1}{2}\left(3\left|\boldsymbol{\beta}_{\|}\right|^{2}-|\boldsymbol{\beta}|^{2}\right)\left[D_{0}\left(x_{\nu}\right)+D_{1}\left(x_{\nu}\right) \Theta\right]\right\} \mathrm{d} \mathbf{r}_{\|},
\end{aligned}
$$

where $\boldsymbol{\beta}(\equiv \mathbf{v} / c)$ and $\boldsymbol{\beta}_{\|}\left(\equiv \mathbf{v}_{\|} / c\right)$ are the parameterized gas velocities relative to the peculiar veloc- 
Table 1

Initial merger parameters

\begin{tabular}{cccccc}
\hline \hline & $M_{1}\left(M_{\odot}\right)$ & $\xi$ & $V\left(\mathrm{~km} \mathrm{~s}^{-1}\right)$ & $P\left(h_{70}^{-1} \mathrm{kpc}\right)$ & $\left(f_{\mathrm{b} 1}, f_{\mathrm{b} 2}\right)$ \\
\hline Model A & $1.3 \times 10^{15}$ & 2.0 & 3000 & 300 & $(0.10,0.10)$ \\
Model B & $2.5 \times 10^{15}$ & 3.6 & 2500 & 800 & $(0.05,0.10)$ \\
Extended Model B & $2.5 \times 10^{15}$ & 3.6 & 2500 & 800 & $(0.11,0.12)$ \\
\hline \hline
\end{tabular}

ity of the center of mass of the cluster, $\mathbf{r}_{\|} /\left|\mathbf{r}_{\|}\right|$is the unit vector of the line of sight directing away from us; $Y_{0}\left(x_{\nu}\right), Y_{1}\left(x_{\nu}\right), C_{1}\left(x_{\nu}\right), C_{2}\left(x_{\nu}\right), D_{0}\left(x_{\nu}\right)$, and $D_{1}\left(x_{\nu}\right)$ are the coefficients for the polynomial approximation of the relativistic correction (see eqs. 24-32 in Nozawa et al. 1998). Since the analytic expression of the kSZ effect (i.e., Eq. 2) can provide a convergent result in our study, we simply adopt this form for the calculation. Note here that Equation (2) does not involve the kSZ effect induced by the peculiar motion of the center of mass of the cluster. Lindner et al. (2015) presents a best-fit peculiar velocity of ACT-CL J0102-4915 as $v_{p}=-1100_{-2200}^{+1300}$ (or $-2800_{-3100}^{+1700}$ ) $\mathrm{km} \mathrm{s}^{-1}$ by using three (or five) bands observations of the SZ spectra. However, in this work we still assume that the center of mass of the entire merging system is at rest in the CMB rest frame (i.e., $v_{p}=0$ ), for the following reasons. (1) The main scientific goal of this paper is to investigate and predict the observational features of the gas motion caused by the merging process. (2) The observational constraints on $v_{p}$ still have large uncertainties (see Lindner et al. 2015). (3) We find that the $\mathrm{kSZ}$ effect $\mathcal{R}\left(v_{p}, x_{\nu}, \Theta\right)$ induced by the peculiar velocity of the cluster could be well modeled by

$\left.\mathcal{R}\left(v_{p}, x_{\nu}, \Theta\right) \simeq \kappa \cdot \frac{v_{p}}{1000 \mathrm{~km} \mathrm{~s}^{-1}} \cdot \frac{\Delta T}{T_{\mathrm{CMB}}}\right|_{\mathrm{tSZ}} ^{\nu=148 \mathrm{GHz}}$,

where $\kappa=0.085$ for both of models $\mathrm{A}$ and $\mathrm{B}$, which does not significantly depend on $\nu$ within the frequency range we are interested in. Thus, it could be easily separated from the other SZ components.

- The CMB temperature changes caused by the RS effect could be approximated as

$$
\left.\frac{\Delta T}{T_{\mathrm{CMB}}}\right|_{\mathrm{RS}}=\frac{4 G}{c^{3}} \int \mathrm{d}^{3} \mathbf{r}^{\prime} \rho_{\mathrm{tot}}\left(\mathbf{r}^{\prime}\right) \mathbf{v}\left(\mathbf{r}^{\prime}\right) \cdot \frac{\mathbf{r}_{\perp}-\mathbf{r}_{\perp}^{\prime}}{\left|\mathbf{r}_{\perp}-\mathbf{r}_{\perp}^{\prime}\right|^{2}},
$$

where $G$ represents the gravitational constant; $\rho_{\text {tot }}(\mathbf{r})$ is the cluster total mass density (see eq. 4 in Rubiño-Martín et al. 2004).

We present the results of the secondary CMB anisotropies at $\nu=148$ and $218 \mathrm{GHz}$ in Section 3.1 to match the frequencies covered by the current high resolution SZ measuring instruments, e.g., ACT, South Pole Telescope (SPT).

We model the X-ray spectra of ACT-CL J0102-4915 by

$$
S_{\mathrm{X}}=\frac{1}{4 \pi(1+z)^{3} \Delta \nu_{0}} \int n_{\mathrm{e}}(\mathbf{r}) n_{\mathrm{H}}(\mathbf{r}) \Lambda_{\nu}\left(T_{\text {gas }}, Z\right) \mathrm{d} \nu_{0} \mathrm{~d} \mathbf{r}_{\|},
$$

where $\Lambda_{\nu}\left(T_{\text {gas }}, Z\right)$ is the cooling function at the frequency $\nu=\nu_{0} \sqrt{\left(1+\left|\boldsymbol{\beta}_{\|}\right|\right) /\left(1-\left|\boldsymbol{\beta}_{\|}\right|\right)}$, obtained by adopting the MEKAL model in the XSPEC v12.8 package ${ }^{1}$. Here $n_{\mathrm{H}}(\mathbf{r}) \equiv 0.83 n_{\mathrm{e}}(\mathbf{r})$ is the number density of the hydrogen; the spectral resolution $\Delta \nu_{0}$ is set to $5 \mathrm{eV}$. We further assume a uniform metallicity distribution $\left(Z=0.3 Z_{\odot}\right)$ of the cluster in the calculation, where the solar metal abundance $Z_{\odot}$ is adopted from Anders \& Grevesse (1989). In this study, we are particularly interested in the H-like $(\sim 7.0 \mathrm{keV})$ and He-like $(\sim 6.7 \mathrm{keV})$ Fe-K lines emitted from the hot ICM. To model the thermal broadening of the spectral lines due to the thermal motion of the ions, we simply smooth the cooling function by a Gaussian kernel with the full width at half maximum (FWHM) of $4.9 \mathrm{eV}\left(k_{\mathrm{B}} T_{\text {gas }} / 5 \mathrm{keV}\right)^{1 / 2}$ (see eq. 11 in Kitayama et al. 2014). In ACT-CL J0102-4915, the high line-of-sight bulk velocities of the gas play the dominant role in the broadening of the X-ray spectral lines (see Section 3.2).

\section{MODELING RESULTS}

\subsection{The secondary $C M B$ anisotropies}

Figure 1 shows the distributions of the tSZ effect, the kSZ effect, and the RS effect for fiducial models A (top panels) and B (middle panels), and extended model B (bottom panels) at $148 \mathrm{GHz}$. The images have been smoothed by a Gaussian kernel with an FWHM of $10^{\prime \prime}$, which corresponds to the measurement capability of the Atacama Large Millimeter/Submillimeter Array (ALMA). Fiducial model A shows an almost axisymmetric morphology of its secondary $\mathrm{CMB}$ anisotropies with respect to the merger direction, because it is a nearly head-on merger (see Table 1); while the distributions resulting from model B look asymmetric. By comparing the panels in Figure 1, we come to the following conclusions.

- At $148 \mathrm{GHz}$, the tSZ effect dominates the secondary CMB anisotropies resulting from ACT-CL J01024915 , which reveals the hot electron density distributions in the merger system. Fiducial models A and B show a generally similar morphology of the tSZ effect distributions. In model B, the significant tSZ signals extend to a larger radius, because model $\mathrm{B}$ has a higher cluster mass (i.e., $3.2 \times 10^{15} M_{\odot}$ ).

- The kSZ effect traces the motions of the gas component in galaxy clusters. In the middle column of Figure 1, the red-yellow and the blue-cyan colorbars represent the positive (i.e., most of the gas moving towards the observer along the lineof-sight) and the negative (i.e., that moving away from the observer) kSZ effect signals, respectively.

\footnotetext{
1 See http://heasarc.nasa.gov/xanadu/xspec/
} 
The interfaces between the positive and negative signals mark the positions of the shocks or the cold fronts formed during the merger process (see also the temperature distributions in Fig. 6). At $148 \mathrm{GHz}$, the strength of the kSZ effect is around one order of magnitude weaker than that of the tSZ effect in both of the models. Fiducial model $\mathrm{B}$ reveals a twisted morphology of the $\mathrm{kSZ}$ effect, because of its off-axis merger configuration. The maximal strength of the $\mathrm{kSZ}$ effect of model B is approximately three times higher than that of model A. This is mostly due to the higher relative radial velocity between the two merging subclusters in model B (see Fig. 6b, and also section 3.3.5 in ZYL15 for more discussions on the relative radial velocity of ACT-CL J0102-4915). The differences in the morphology of the $\mathrm{kSZ}$ effect between models $\mathrm{A}$ and $\mathrm{B}$ may provide a way to distinguish these two fiducial models in the future SZ observations of ACT-CL J0102-4915.

- The strength of the RS effect is more than 10 times weaker than that of the $\mathrm{kSZ}$ effect for both of models A and B. For the capability of the current SZ measuring instruments (e.g., ACT or SPT), contributions of the RS effect to the secondary CMB temperature anisotropies resulting from ACT-CL J0102-4915 are negligible.

In the Kompaneets approximation, the crossover frequency of the tSZ effect is $\simeq 218 \mathrm{GHz}$, which provides a unique window for detecting the $\mathrm{kSZ}$ signals. However, the relativistic correction for the gas with high temperature contributes non-negligible tSZ signals to the CMB map at $218 \mathrm{GHz}$. The strengths of the thermal and the kinetic SZ effects of ACT-CL J0102-4915 are actually comparable at $218 \mathrm{GHz}$. To extract the kSZ signals, we combine the distributions of the SZ effect at 148 and $218 \mathrm{GHz}$ as follows,

$\left.\frac{\Delta T}{T_{\mathrm{CMB}}}\right|_{\mathrm{res}}=\frac{1}{1-\alpha}\left(\left.\frac{\Delta T}{T_{\mathrm{CMB}}}\right|_{\mathrm{tSZ}+\mathrm{kSz}} ^{218 \mathrm{GHz}}-\left.\alpha \cdot \frac{\Delta T}{T_{\mathrm{CMB}}}\right|_{\mathrm{tSZ}+\mathrm{kSz}} ^{148 \mathrm{GHz}}\right)$,

where $\alpha$ is a parameter selected to maximally remove the contamination of the tSZ effect in the residual image ${ }^{2}$ The best-fit parameter $\alpha$ is 0.143 and 0.154 for fiducial models $\mathrm{A}$ and $\mathrm{B}$, respectively. As a reference, the ratio of the tSZ distortions at $218 \mathrm{GHz}$ and $148 \mathrm{GHz}$ is $\mathcal{F}\left(x_{\nu=218 \mathrm{GHz}}, \Theta\right) / \mathcal{F}\left(x_{\nu=148 \mathrm{GHz}}, \Theta\right)=0.095$ (see Eq. 1), when fixing the gas temperature to the best-fit cluster $\mathrm{X}$-ray temperature $(\sim 15 \mathrm{keV})$. The best-fit parameter $\alpha$ is larger than this value so that some tSZ signal produced by the high-temperature gas can be removed. It is worthy of noting that, in reality, the additional contaminations in the cm-wavelength band (e.g., dusty galaxies, radio point sources) may make the situation more complicated.

The recovered kSZ effect residual maps are presented in Figure 2. The distributions (left panels) are generally in agreement with the models shown in the middle column

\footnotetext{
${ }^{2}$ We obtain the best-fit parameter $\alpha$ by minimizing the summation of $\left(\left.\frac{\Delta T}{T_{\mathrm{CMB}}}\right|_{\mathrm{tSZ}} ^{218 \mathrm{GHz}}-\left.\alpha \cdot \frac{\Delta T}{T_{\mathrm{CMB}}}\right|_{\mathrm{tSZ}} ^{148 \mathrm{GHz}}\right)^{2}$ over all the pixels of the thermal SZ images.
}

of Figure 1, which illustrate that most of the tSZ emissions have been effectively removed after the extracting process. However, the tSZ contamination contributed by the high-temperature gas can still be seen around the cluster shock regions (see also left panels in Fig. 5). By comparing the residual images at different angular resolutions in Figure 2, we find that even though the lowresolution ones (right panels with FWHM $\sim 1.3^{\prime}$ ) lack substructures and high signal contrasts, the differences between fiducial models $\mathrm{A}$ and $\mathrm{B}$ are still distinguishable in these maps. Our results suggest that, to detect the kSZ signal of ACT-CL J0102-4915 in the future, the $\mathrm{CMB}$ measurement sensitivity better than $10^{-5} \mathrm{~K}$ is needed. ALMA should have the capability to reach such a goal (see Basu et al. 2016, Yamada et al. 2012). It may be harder to extract the kSZ effect of ACT-CL J01024915 in reality than in the illustration here, because no further observational contaminations and uncertainties are involved in our models.

Figure 3 shows the SZ spectra of fiducial models A and $\mathrm{B}$, and extended model $\mathrm{B}$ averaged within the white circles indicated in Figure 1. The amplitude of the tSZ spectrum of model A is around $20 \%$ higher than that of model B, because the core of the secondary cluster in model A shows a higher gas temperature (see panels (a) in Fig. 6 below, and also fig. 7 in ZYL15). For both of models $\mathrm{A}$ and $\mathrm{B}$, the integrated strength of the $\mathrm{kSZ}$ spectra is much weaker $(<1 / 20)$ than that of the tSZ spectra. We emphasize here again that the calculation does not consider the $\mathrm{kSZ}$ effect induced by the peculiar velocity of the cluster.

In the last part of this subsection, we discuss the possibility to detect the high-temperature electrons in the shock regions by using the relativistic correction of the tSZ effect. Figure 4 shows the crossover frequency of the tSZ effect as a function of the electron temperature. The crossover frequency increases as the electron temperature becomes higher. At $235 \mathrm{GHz}$, the gas hotter than $40 \mathrm{keV}$ generates negative tSZ signals, but the cooler gas generates the positive ones. The left panels of Figure 5 show the tSZ effect generated by the hot gas $(>40 \mathrm{keV})$ of models $\mathrm{A}$ and $\mathrm{B}$ at $235 \mathrm{GHz}$. The signals coincide with the north-western (NW) and the south-eastern (SE) shocks in positions (see top panels in Fig. 6), and show a similar strength as the kSZ effect (see middle column in Fig. 1). Therefore, we expect that the tSZ effect contributed by the hot electrons in the shock regions of ACT-CL J0102-4915 is negative if the electrons could be efficiently heated by the ions in reality. Although being challenging, the following points should be considered to detect this feature in future observations: (1) the electrons are heated to high temperatures around the shock fronts, which needs high-resolution observations $\left(\lesssim 10^{\prime \prime}\right)$ to resolve; (2) the tSZ effect contributed by the relativistic correction partly overlaps with the kSZ emission, and they both show the negative temperature deviation and need to be isolated properly; and (3) the strength of the relativistic correction of the tSZ effect is sensitive to the frequency. Only the measurement within a narrow frequency band is expected to directly see the signal. The right panels of Figure 5 show the mock SZ measurements of models $\mathrm{A}$ and $\mathrm{B}$ within a finite band width 

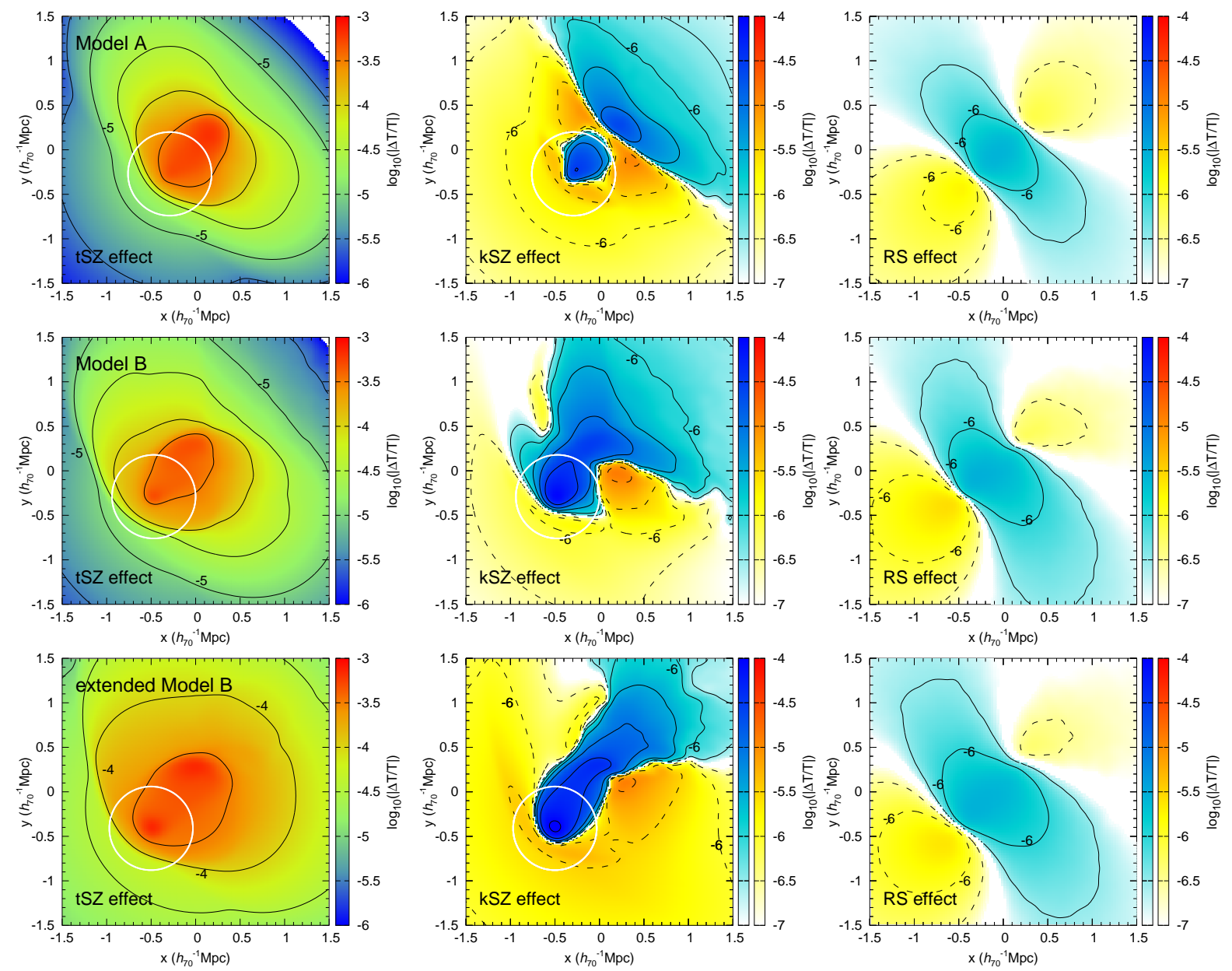

Figure 1. The distributions of the tSZ effect, the kSZ effect, and the RS effect resulting from a merging galaxy cluster with the merging configurations in fiducial model A (top panels), fiducial model B (middle panels), and extended model B (bottom panels). All the maps are estimated in the $148 \mathrm{GHz}$ band, and smoothed by a Gaussian kernel with an FWHM of $10^{\prime \prime}$. In the second and the third columns, the red-yellow colorbar (dashed black contour lines) and the blue-cyan colorbar (solid black contour lines) represent the positive and the negative CMB temperature variations, respectively. In all the panels, the interval between two successive contour levels with the same line types is 0.5 in $\log _{10}(|\Delta T / T|)$. The SZ surface brightness averaged within the white circles (with the center being at the position of the maximum of the X-ray emission and the radius being $1^{\prime}$ ) are shown in Figure 3 This figure shows a significant difference between models $\mathrm{A}$ and $\mathrm{B}$ in the morphology of the kSZ effect distributions (see Section 3.1).

$\nu \in\left[\nu_{\min }, \nu_{\max }\right]$, i.e., $\mathcal{F}$ in Equation $(1)$ is replaced by

$$
\mathcal{F}\left(x_{\left[\nu_{\min }, \nu_{\max }\right.}, \Theta\right)=\frac{\int_{\nu_{\min }}^{\nu_{\max }} \mathcal{F}\left(x_{\nu^{\prime}}, \Theta\right) \mathrm{d} \nu^{\prime}}{\nu_{\max }-\nu_{\min }},
$$

where $\nu_{\min }=230 \mathrm{GHz}$ and $\nu_{\max }=240 \mathrm{GHz}$ (see Eq. 1). The kSZ effect is estimated at $235 \mathrm{GHz}$, since it does not significantly depend on the frequency within this band (see Fig. 3). By comparing them with the corresponding left panels, we can see the similar pattern of the negative SZ signal near the shock regions. The signals become insignificant if we broaden the band width.

\subsection{The X-ray spectra}

In this section, we present a detailed investigation into the broadening and the shifting of the X-ray spectral lines of ACT-CL J0102-4915, due to the gas motion. Before that, we examine the velocity fields of models A and $\mathrm{B}$ to gain a sense of motions of the ICM caused by the cluster merging process. Panels (a), (b), and (c) in Figure 6 show the spectroscopic-weighted temperature, line-of-sight velocity $\left(\mu_{||}\right)$, and line-of-sight velocity dispersion $\left(\sigma_{\|}\right)$distributions for fiducial models $\mathrm{A}$ and $\mathrm{B}$, and extended model B, respectively. The spectroscopicweighted temperature is defined as

$$
T\left(\mathbf{r}_{\perp}\right)=\frac{\int w(\mathbf{r}) T_{\mathrm{gas}}(\mathbf{r}) \mathrm{d} \mathbf{r}_{\|}}{\int w(\mathbf{r}) \mathrm{d} \mathbf{r}_{\|}}
$$

where $w(\mathbf{r})=\rho_{\text {gas }}^{2}(\mathbf{r}) T_{\text {gas }}^{-3 / 4}(\mathbf{r})$ is taken as a good approximation for the projected spectroscopic temperature of clusters obtained from the X-ray observations (Mazzotta et al. 2004). In panels (a), we can see the NW and SE shocks in both of the models. The temperature of the ambient gas has been shock-heated to around $30-50 \mathrm{keV}$. Most of the substructures revealed in the temperature distributions are also seen in the velocity maps (see panels b and c), because the shocks are formed when the gas flows are colliding with high relative velocities. Compared with the shocks in model A, those in model B are stronger and more asymmetric, because model $\mathrm{B}$ has a higher merger mass and a larger impact parameter. The shocks match the double radio relics of ACT-CL J01024915 in position (see figs. 1 and 2 in Lindner et al. 2014). This might be explained by that the observed radio relics 

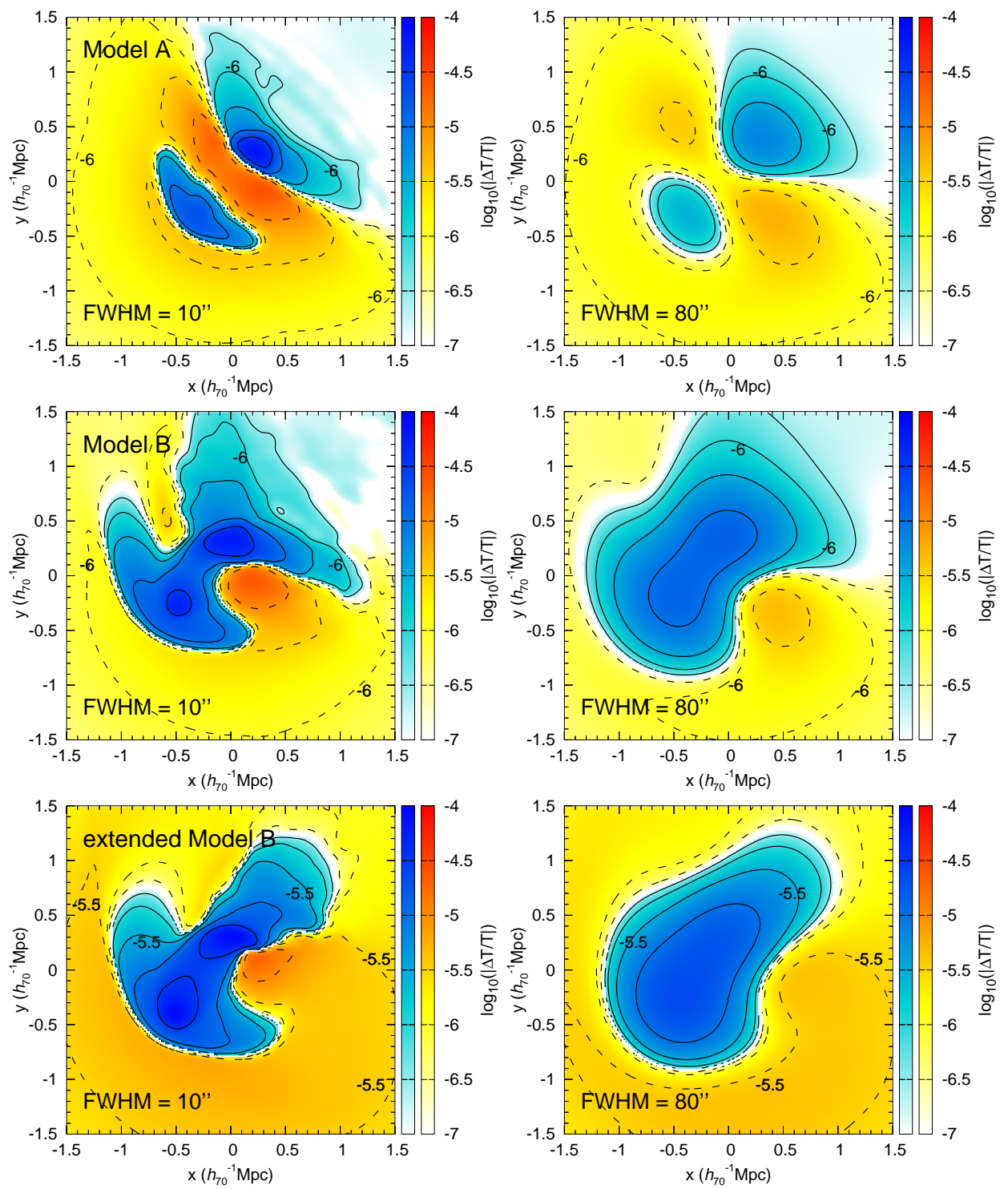

Figure 2. Legends similar to those in Figure 1 but for the extracted kSZ effect distributions by combining the SZ maps at 148 and $218 \mathrm{GHz}$ (see Eq. 6). The results are smoothed by a Gaussian kernel with FWHM $=10^{\prime \prime}$ (left panel) and FWHM=80" (right panel), respectively. This higure indicates that the measuring sensitivity to detect the kSZ signals of ACT-CL J0102-4915 and distinguish between the proposed merging configurations needs to be better than $10^{-5} \mathrm{~K}$ level (see Section 3.1 .

is via first-order Fermi acceleration by shocks (Lindner et al. 2014).

Panels (b) and (c) illustrate the gas velocity distributions in these two models. The line-of-sight velocity and velocity dispersion are defined as

$$
\begin{aligned}
\mu_{\|}\left(\mathbf{r}_{\perp}\right) & =\frac{\int \epsilon(\mathbf{r}) \mathbf{v}_{\|}(\mathbf{r}) \mathrm{d} \mathbf{r}_{\|}}{\int \epsilon(\mathbf{r}) \mathrm{d} \mathbf{r}_{\|}}, \\
\sigma_{\|}^{2}\left(\mathbf{r}_{\perp}\right) & =\frac{\int \epsilon(\mathbf{r})\left[\mathbf{v}_{\|}(\mathbf{r})-\mu_{\| \mid}\left(\mathbf{r}_{\perp}\right)\right]^{2} \mathrm{~d} \mathbf{r}_{\|}}{\int \epsilon(\mathbf{r}) \mathrm{d} \mathbf{r}_{\|}},
\end{aligned}
$$

respectively, where the weight function adopts the Xray emissivity $\epsilon(\mathbf{r})=n_{\mathrm{e}}(\mathbf{r}) n_{\mathrm{H}}(\mathbf{r}) \int \Lambda_{\nu}\left(T_{\text {gas }}, Z\right) \mathrm{d} \nu$ covering $0.5-7.5 \mathrm{keV}$. As expected, the regions of the secondary gas cores show high positive line-of-sight velocities (i.e., bulk velocities), which spatially coincide with the cold fronts shown in the temperature distributions (i.e., region 2 marked in the panels). The features shown in panels (b) are similar with those viewed in the kSZ effect distributions (see the middle column in Fig. 1), because the amplitude of the $\mathrm{kSZ}$ effect is proportional to $\mu_{\|}$in the first-order approximation. In panels (c), the high line-of-sight velocity dispersions $\left(500-1500 \mathrm{~km} \mathrm{~s}^{-1}\right)$ arise from (1) the turbulence in the ICM generated during the merger, or (2) the projection of the gas structures with different velocities along the line-of-sight. In particular, the gas trailing after the secondary clusters in both of models $\mathrm{A}$ and $\mathrm{B}$ (i.e., around region 4 marked in the panels) shows high velocity dispersions $\left(\sim 1500 \mathrm{~km} \mathrm{~s}^{-1}\right)$. The reason is that the multiple shocks propagating in different directions significantly disturb the gas distributions in these areas.

Panels (d) in Figure 6 show the X-ray spectra extracted from the fields within the white squares indicated in the other panels of Figure 6. The spectra are presented in the cluster rest frame. For each model, we set four squares 


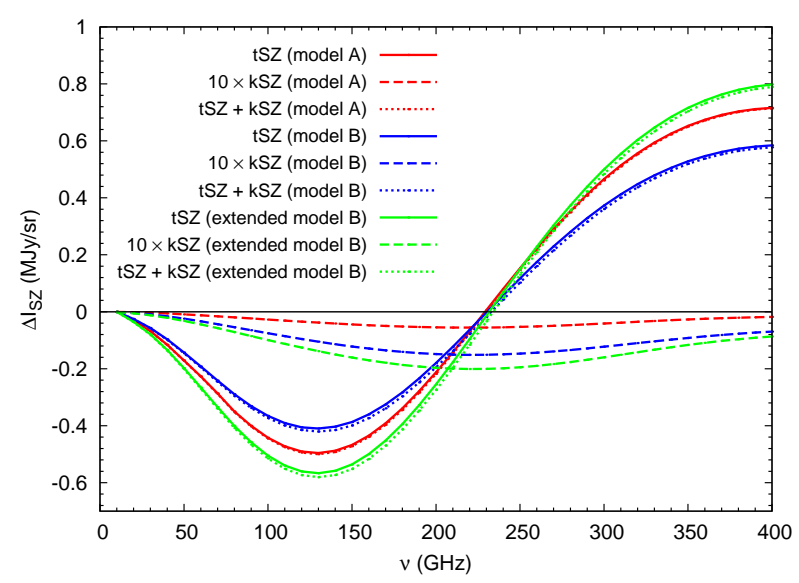

Figure 3. Spectral distortion of the CMB radiation due to the tSZ effect, the kSZ effect, and their combination. The vertical axis shows the average intensity distortion within the white circles marked in Figure 1 To present a clear comparison with the tSZ spectra, we show the kSZ spectra amplified by a factor of 10 as indicated in this figure. The strength of the $\mathrm{kSZ}$ spectra is around $1 / 30-1 / 20$ of that of the tSZ spectra. See details in Section 3.1.

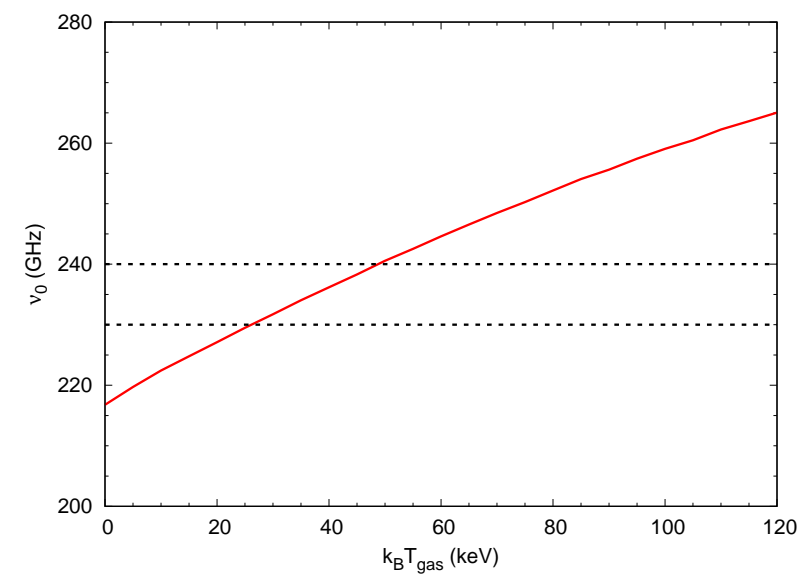

Figure 4. Crossover frequency of the tSZ effect, $\nu_{0}$, as a function of the gas temperature. The frequency band $\nu \in$ $[230 \mathrm{GHz}, 240 \mathrm{GHz}]$ adopted in the right panels of Figure 5 is marked by the horizontal dashed lines. The crossover frequency shifts to a larger value when the electron temperature gets higher. See Sections 2.2 and 3.1

to cover the region in front of the SE shock (region 1), the brightest X-ray emission core (region 2), the wakelike structure (region 3), and the high velocity dispersion region (region 4 ), respectively. The Doppler shifting and broadening of the spectral lines are expected to contain the information on the line-of-sight motion of the gas in the ICM. From the panels, we find the following main points.

- In both of models A and B, the lines emitted from regions 3 and 4 are significantly broadened, which includes the effects of the thermal broadening and the Doppler broadening (also known as the kinetic broadening). The relative contribution of these two mechanisms to the line broadening can be approximated by

$$
\frac{W_{\text {therm }}}{W_{\text {kin }}} \simeq \frac{\sqrt{k_{\mathrm{B}} T_{\text {gas }} / 5 \mathrm{keV}}}{\sigma_{\|} / 10^{2} \mathrm{~km} \mathrm{~s}^{-1}},
$$

where $W_{\text {therm }}$ and $W_{\text {kin }}$ are the FWHMs of the thermal and the kinetic broadenings, respectively (see eqs. 8, 11-12 in Kitayama et al. 2014). The typical velocity dispersion and the gas temperature in regions 3 and 4 are $\sim 10^{3} \mathrm{~km} \mathrm{~s}^{-1}$ and $\sim 30 \mathrm{keV}$, respectively. So the kinetic mechanism plays the dominative role in broadening the spectral lines in ACT-CL J0102-4915. For example, the FWHM of the H-like Fe-K line emitted from the twin-tailed structure of the cluster (in region 3, see Fig. 6) is around $50 \mathrm{eV}$. In addition, we do not see remarkable He-like Fe-K lines emission from regions 3 and 4 in both of the models, because of the generally high gas temperature in these areas.

- In fiducial model A, we can see a significant line shift $(\Delta E \sim 25 \mathrm{eV})$ between the H-like Fe-K lines emitted from region 2 and region 3 , which approximately corresponds to $1000 \mathrm{~km} \mathrm{~s}^{-1}$ in velocity. This shift is mainly caused by the relative radial velocity between the primary and the secondary clusters in model A. However, in model B, we do not find a similar feature. The reason is that, the elongated gas distribution of the secondary cluster in model B covers most of regions 2 and 3, which holds a similar bulk velocity. The high-resolution spectroscopic measurement in the future are expected to detect the line shifts among the different regions in ACT-CL J0102-4915, and further provide a possible constraint on the merger configuration of the cluster.

The Soft X-ray Spectrometer (SXS) on XARM can provide an energy resolution of $<7 \mathrm{eV}$, which is expected to detect the modeled line features shown in Figure 6(d) (see Takahashi et al. 2012). Based on our simulations, we simply estimate the required exposure time to identify the Fe-K lines near the cluster X-ray center by using the properties of SXS instrument on ASTRO-H (i.e., angular resolution $\sim 1.3^{\prime}$, effective area $\left.\sim 200 \mathrm{~cm}^{2}\right)$. It approximately needs $\sim 200 \mathrm{ks}$ to collect 100 photons for each Fe-K line (i.e., the peaks shown in the blue lines in panels (d) of Fig. 6). The ATHENA would further provide high spatial resolution measurements but also with more than 10 times larger effective area (Nandra et al. 2013). However, it is worthy of noting that we assume a universal metallicity $Z=0.3 Z_{\odot}$ for both of the models in this study. But in reality, ACT-CL J0102-4915 reveals a higher central metal abundance of $0.57 \pm 0.20$ (Menanteau et al. 2012). This spatially non-uniform metal distribution may have impacts on the shape and the amplitude of the X-ray spectral lines.

\section{CONCLUSIONS}

In this work, we predict the $\mathrm{kSZ}$ effect, relativistic correction of the $\mathrm{tSZ}$ effect, and the X-ray spectra and line emission of ACT-CL J0102-4915, based on fiducial models A and B proposed in ZYL15. The kSZ effect and the broadening and the shifting of the spectral lines are expected to provide insights into the gas motions of the ICM, which include the bulk velocities of the merging subclusters and the turbulence produced by the merger shocks. Our findings are concluded as follows.

- The amplitudes of the kSZ effect resulting from 

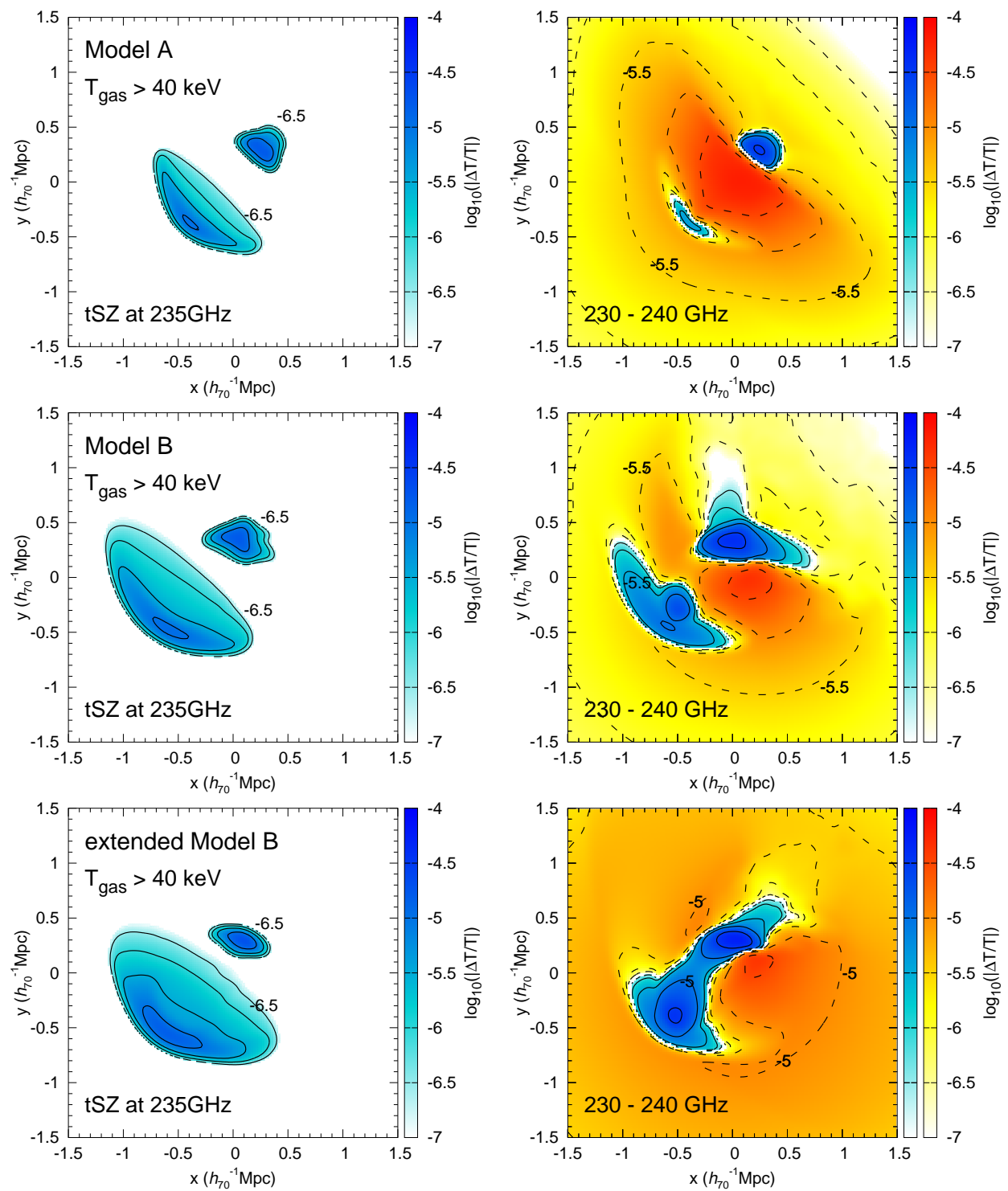

Figure 5. Left panels: tSZ effect generated by the high temperature gas ( $\left.T_{\text {gas }}>40 \mathrm{keV}\right)$ at $235 \mathrm{GHz}$, which is smoothed by a Gaussian kernel with FWHM $=10^{\prime \prime}$ (the same as the right panels). Right panels: mock SZ observations within the frequency band $\nu \in[230 \mathrm{GHz}, 240 \mathrm{GHz}]$ (see Eq. 7). This figure illustrates the possibility to detect the high temperature electrons heated by the shocks during the merger by using the relativistic correction of the tSZ effect.

fiducial models $\mathrm{A}$ and $\mathrm{B}$ are both at an order of $\Delta T / T \sim 10^{-5}$ (the kSZ effect induced by the peculiar velocity of the entire cluster is not included). The morphologies of the kSZ effect resulting from these two models are different, and the difference indicate the different line-of-sight velocity distributions in these two models. Model A shows an almost axisymmetric morphology of the kSZ effect; but model B shows a twisted one because of the offaxis merger configuration. The maximal strength of the $\mathrm{kSZ}$ effect of model B is roughly three times higher than that of model A. The kSZ measurements thus provide a unique way to figure out the merger scenario of ACT-CL J0102-4915. Though the $\mathrm{kSZ}$ effect is as weak as $\Delta T / T \sim 10^{-5}$, we find that combining the SZ distributions at $148 \mathrm{GHz}$ and $218 \mathrm{GHz}$ could robustly extract the kSZ signals from the strong tSZ emissions. The CMB instruments reaching $\mu \mathrm{K}$ sensitivity may detect the $\mathrm{kSZ}$ signals of ACT-CL J0102-4915 in the future.

- Both models A and B produce negative SZ temperature deviations around the shock regions at $\sim 230-250 \mathrm{GHz}$, due to the relativistic correction of the tSZ effect. These can be used to detect the possible high temperature electrons heated by the shocks during the cluster merger, which is important to give a constraint on the timescale of the thermal equilibrium between the ions and the electrons.

- Both models A and B reveal the shifting and broadening of the X-ray spectral lines, which could be used to determine the velocity fields of the merging cluster. The FWHM of the H-like Fe-K line emitted from the twin-tailed structure of the cluster (in region 3, see Fig. 6) is around $50 \mathrm{eV}$, mainly caused by the kinetic broadening mechanism. The shifting 

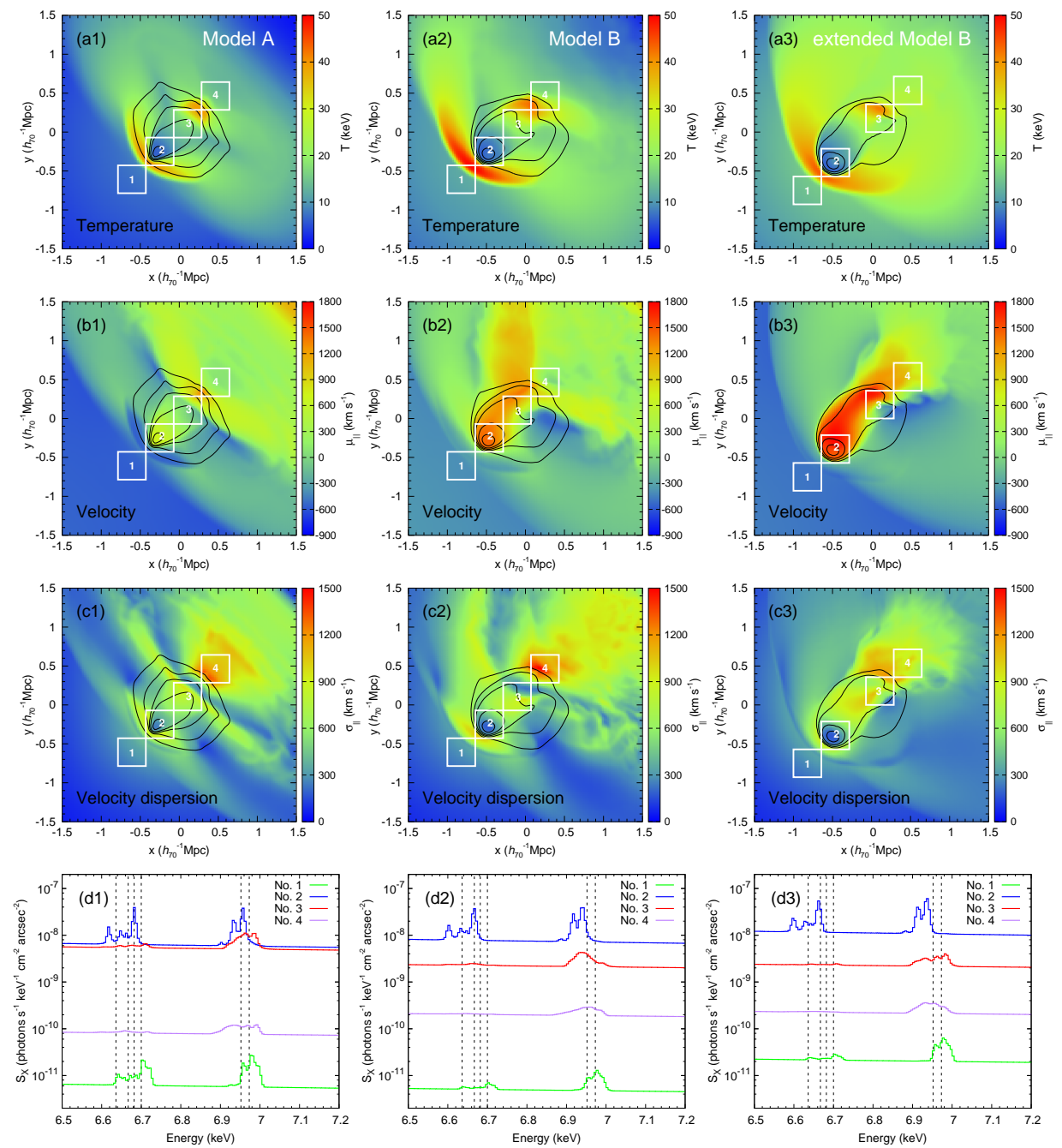

Figure 6. Distributions of the spectroscopic-weighted temperature (panels a), the line-of-sight velocity $\mu_{\|}$(panels b), the line-of-sight velocity dispersion $\sigma_{\|}$(panels c), and the X-ray spectra (panels d) extracted from the corresponding white square regions indicated in panels (a)-(c) for fiducial model A (left column), fiducial model B (middle column), and extended model B (right column). The spectra are presented in the cluster rest frame. The overlaid black contours in panels (a), (b), and (c) represent the X-ray surface brightness. The contour level starts with $10^{-8.5}$ photons s${ }^{-1} \mathrm{~cm}^{-2} \operatorname{arcsec}^{-2}$ and increases by a factor of $10^{0.5}$ from outside to inside. As a reference, we mark the peak positions of the dominant H-like and He-like Fe-K lines in the rest frame in panels (d) by the vertical dashed lines. There is no remarkable He-like Fe-K lines emitted from regions 3 and 4 in both of the models, because of the generally high gas temperature in these areas. This figure illustrates the remarkable shifting and the broadening of the X-ray spectral lines of the models (see Section 3.2 .

of the X-ray spectral lines caused by the gas motion is different in the two models, which can be up to $25 \mathrm{eV}$ in some cluster regions. In fiducial model A, we see a line shift between those emitted from the brightest X-ray emission core (region 2, see Fig. 6) and from the twin-tailed structure. However, we do not see the same feature in model B, because the tidally elongated gas distribution of the secondary cluster in model B covers most part of the sampling regions.

We conclude that the future observational instruments for the kSZ effect (e.g., ACT, ALMA) or the X-ray spectral lines (e.g., XARM, ATHENA) may be able to detect the gas motions in ACT-CL J0102-4915, and give a constraint on the cluster merger configurations.

We thank Daisuke Nagai, Erwin Lau, David Spergel, Rashid Sunyaev, and Eugene Churazov for suggestions and discussions. This work was supported in part by the National Natural Science Foundation of China under nos. 11673001, 11273004, 11373031, 11390372, the National Key R\&D Program of China (Grant Nos. 2016YFA0400703, 2016YFA0400704).

\section{REFERENCES}

Anders, E., \& Grevesse, N. 1989, Geochim. Cosmochim. Acta, 53, 197

Basu, K., Sommer, M., Erler, J., et al. 2016, ApJ, 829, L23

Benson, B. A., Ade, P. A. R., Ahmed, Z., et al. 2014, Proc. SPIE, 9153, 91531P

Biffi, V., Dolag, K., Böhringer, H. 2013, MNRAS, 428, 1395

Birkinshaw, M. 1999, Phys. Rep., 310, 97

Fryxell, B., Olson, K., Ricker, P., et al. 2000, ApJS, 131, 273

Hand, N., Addison, G. E., Aubourg, E., et al. 2012, Physical

Review Letters, 109, 041101

Itoh, N., Kohyama, Y., \& Nozawa, S. 1998, ApJ, 502, 7

Jee, M. J., Hughes, J. P., Menanteau, F., et al. 2014, ApJ, 785, 20

Kitayama, T., Bautz, M., Markevitch, M., et al. 2014,

arXiv:1412.1176 
Lindner, R. R., Baker, A. J., Hughes, J. P., et al. 2014, ApJ, 786, 49

Lindner, R. R., Aguirre, P., Baker, A. J., et al. 2015, ApJ, 803, 79 Marriage, T. A., Acquaviva, V., Ade, P. A. R., et al. 2011, ApJ, 737, 61

Mazzotta, P., Rasia, E., Moscardini, L., \& Tormen, G. 2004, MNRAS, 354, 10

Menanteau, F., Hughes, J. P., Sifón, C., et al. 2012, ApJ, 748, 7

Mroczkowski, T., Dicker, S., Sayers, J., et al. 2012, ApJ, 761, 47

Nandra, K., Barret, D., Barcons, X., et al. 2013, arXiv:1306.2307

Nozawa, S., Itoh, N., \& Kohyama, Y. 1998, ApJ, 508, 17

Nozawa, S., Itoh, N., Kawana, Y., \& Kohyama, Y. 2000, ApJ, 536,31

Planck Collaboration, Ade, P. A. R., Aghanim, N., et al. 2016, A\&A, 586, A140

Ricker, P. M. 2008, ApJS, 176, 293

Rubiño-Martín, J. A., Hernández-Monteagudo, C., \& Enßlin, T. A. 2004, A\&A, 419, 439
Russell, H. R., McNamara, B. R., Sanders, J. S., et al. 2012, MNRAS, 423, 236

Sayers, J., Mroczkowski, T., Zemcov, M., et al. 2013, ApJ, 778, 52

Sayers, J., Zemcov, M., Glenn, J., et al. 2016, ApJ, 820, 101

Schaan, E., Ferraro, S., Vargas-Magaña, M., et al. 2015, arXiv: 1510.06442

Soergel, B., Flender, S., Story, K. T., et al. 2016, arXiv:1603.03904

Springel, V., Yoshida, N., \& White, S. D. M. 2001, New A, 6, 79 Sunyaev, R. A., Norman, M. L., \& Bryan, G. L. 2003, Astronomy Letters, 29, 783

Takahashi, T., Mitsuda, K., Kelley, R., et al. 2012, Proc. SPIE, 8443, 84431Z

Yamada, K., Kitayama, T., Takakuwa, S., et al. 2012, PASJ, 64, 102

Zhang, C., Yu, Q., \& Lu, Y. 2015, ApJ, 813, 129 